\title{
Diabetes, Sports and Exercise
}

\author{
Authors \\ Katrin Esefeld1, 4, Stephan Kress'2, 7, Meinolf Behrens'2, 8, Peter Zimmer², Michael Stumvoll3, Ulrike Thurm², \\ Bernhard Gehr2, 9, Christian Brinkmann'2, 5, 6, Martin Halle1, 2, 4
}

\section{Affiliations}

1 Department of Preventive Sports Medicine and Sports Cardiology, Technical University Munich, Munich, Germany

2 Diabetes, Sport and Physical Activity Working Group of the DDG, Germany

3 Medical Department III - Endocrinology, Nephrology, Rheumatology, University Hospital Leipzig, Leipzig, Germany

4 German Center for Cardiovascular Research (Deutsches Zentrum für Herzkreislaufforschung - DZHK), partner site Munich Heart Alliance (MHA), Munich, Germany

5 Institute of Cardiovascular Research and Sport Medicine, German Sport University Cologne, Cologne, Germany

6 IST University of Applied Sciences Düsseldorf, Düsseldorf, Germany

7 Department of Internal Medicine I, Vinzentius Hospital Landau, Germany

8 Diabetes Center Minden, Minden, Germany

9 m\&i specialized clinic Bad Heilbrunn, Germany

published online $\quad 21.12 .2020$

Bibliography

Exp Clin Endocrinol Diabetes 2021; 129: S52-S59

DOI $10.1055 / \mathrm{s}-1284-6097$

ISSN 0947-7349

(c) 2020. Thieme. All rights reserved.

Georg Thieme Verlag KG, Rüdigerstraße 14,

70469 Stuttgart, Germany
German Diabetes Association: Clinical Practice Guidelines

This is a translation of the DDG clinical practice guideline

published in Diabetologie 2020; 15: S148-S155,

DOI 10.1055/a-1194-1638

\section{Correspondence}

Dr. med. Stephan Kress

Vinzentius Hospital, Certified Diabetes Center

Cornichonstraße 4

76829 Landau

Germany

s.kress@vinzentius.de

Prof. Dr. med. Martin Halle

Technical University of Munich, Klinikum rechts der Isar,

Department of Preventive Sports Medicine and Sports

Cardiology

Georg-Brauchle-Ring 56

80992 Munich

Germany

martin.halle@mri.tum.de
For all forms of diabetes mellitus, exercise is one of the most important measures for maintaining good health. Sport and exercise trigger adaptation and repair mechanisms in various organ systems and cells, such as the muscles, nerves, vessels, immune system or brain, which can help ward off diseases [1-4].

There is no need for maximum physical exercise. Exertion according to the motto "running/walking without panting" already brings about good health benefits. According to the recommendations of the American Diabetes Association (ADA) from 2020, adults with diabetes should exercise for 150 minutes/week or more at moderate intensity. Younger and physically fit patients can also exercise more intensively (minimum recommendation: 75 minutes/ week). Exercise should be performed on at least 3 days, with no more than 2 consecutive days of inactivity. Strength training 2-3 times a week is also recommended, but not on consecutive days. Periods of sitting should be interrupted every 30 minutes. For older people, flexibility and balance training is also recommended 2-3 times a week.

Alternative sports such as yoga and tai-chi may also be suitable. For the treatment of obesity, 200-300 minutes of exercise per week and an energy deficit of about 500-750 kcal/day should be aimed for [5].

This practice guideline is intended to outline therapeutic options for physical activity in the treatment of diabetes mellitus and to provide practical recommendations for implementation in type 1 and type 2 patients.

\section{Physiology of the Muscular Glucose Metabolism}

Contractions of the peripheral skeletal muscles increase muscular energy consumption. Depending on the intensity and duration of the exertion, the energy consumption can increase up to the 8-10-fold 
amount of the resting expenditure. If the energy consumption under resting conditions is by a larger amount covered by the oxidation of free fatty acids, the energy requirement under physical exertion is covered increasingly by glycolysis and, in the case of prolonged muscular activity, additionally by beta oxidation of free fatty acids.

When physical exertion begins, intramuscular glucose, which comes from the breakdown of the muscular glycogen reserves, is consumed first. In addition, glucose reaches the muscle cell by increasing the transmembrane glucose transport from the blood. This is achieved by a translocation of glucose transporters, in the case of muscular GLUT-4 from intracellular locations to the muscle cell membrane, a process that is insulin-independent and is induced by muscle contraction. The contraction of the muscle fibers thus corresponds to the physiological effect of insulin. Thus, even in cases of insulin resistance such as type 2 diabetes, an increase in transmembrane glucose transport can be enabled and a reduction in glucose levels can be induced [6].

The systemic glucose decrease resulting from muscular activity is compensated by a precise and adequate increase in hepatic glucose release when no simultaneous glucose reabsorption from food is available. The increase in this release is mainly caused by an inhibition of pancreatic insulin secretion and the resulting drop in insulin levels in the portal vein blood. The contra-insulinary hormones (glucagon, catecholamines and cortisol) have a supporting and modulating effect. If hormones such as glucagon are absent after cell death, as in pancreatitis, this counter-regulation is suspended and this can lead to severe, sometimes fatal, hypoglycemia.

These hepatic and muscular energy stores are replenished during and after muscular activity is completed. Depending on the degree of emptying, glucose uptake into the muscles can be increased for up to 48 hours after the end of muscular activity, which is important for the adjustment of medication and reduction of the insulin dose. Intensive and prolonged muscular exertion such as extended forms of exercise (e.g. walking downhill for several hours while hiking) lead to a strong emptying of the muscular storage. At the same time, this form of exercise can damage the muscle membrane including the insulin receptors, which is reflected in increased insulin resistance and protracts replenishment of the muscular glucose stores.

\section{Benefits and Challenges in the Context of Physical Activity in Type 1 Diabetes}

\section{Pathophysiology}

In type 1 diabetes patients, pancreatic insulin secretion is absent and must be substituted exogenously. With each insulin injection, there is a relative excess of insulin, which increases muscular glucose uptake but blocks hepatic glucose release. This results in a drop in blood glucose levels, which is desirable when the initial serum level is elevated, but which quickly leads to undesirable hypoglycemia if physical activity is prolonged and normoglycemia already exists at the beginning of muscular activity. For this reason, the exact balance of increased exercise and reduced insulin dose is of central importance.

Problems can also occur when a lack of basal insulin has been prevalent for a while and physical activity is performed, because under resting conditions without insulin, glucose uptake into the peripheral muscles is hardly possible. The omission of insulin injections or catheter occlusions during insulin pump therapy lead to absolute insulin deficiency, infections to relative insulin deficiency. This induces an increase in contra-insulinary hormones with an increase in hepatic glucose release. Since the uptake of glucose into the muscles is simultaneously restricted, the serum glucose level rises. Due to the reduced availability of intramuscular glucose, the energy requirement of the working muscles is then primarily covered by free fatty acids, which explains the development of ketoacidosis due to insulin deficiency during muscular activity.

\section{Consequences for physical activity in type 1 diabetes}

- Sport and play are part of the quality of life for everyone, and especially for children and adolescents, an important socially integrative element that should also be promoted for type 1 diabetic patients.

- Regular physical activity also plays an important role in improving the cardiovascular risk profile in type 1 diabetes patients [7].

- However, physical activity and training lead to increased glucose fluctuations during and after physical exercise.

- Physical training can be recommended without restriction if insulin injection, glucose intake through food and energy conversion through physical activity are precisely coordinated. Thus, regular physical activity, as uniformly and daily as possible, can induce metabolic effects and thus optimize glucose regulation in the long term, while sporadic exertion may lead to metabolic fluctuations, especially in untrained and inexperienced patients.

- Due to the individual differences in the response of 1) the muscular glucose metabolism to physical exertion, 2) the glucose increase after food intake, 3 ) the insulin reaction after injection as well as 4) the training status of the muscles and the whole organism, practical knowledge transfer and training including the determination of the individual adaptation is of central importance [8].

- In $>$ Fig. 1 the different intensities of physical exertion (aerobic, mixed aerobic-anaerobic and anaerobic) are shown with the respective influence on the blood glucose level. (Prolonged) exercise in the aerobic intensity range primarily induces hypoglycemia if the insulin dose or carbohydrate intake is not adjusted. Classic aerobic sports are walking, hiking, cycling, swimming, cross-country skiing, etc., which are performed with low to moderate intensity over a prolonged period of time. In contrast, intensive anaerobic activities, such as sprints, usually result in an increase in blood glucose due to the release of catecholamines. Team sports are characterized by an alternation of low, moderate and high intensity.

In most cases, a stable blood glucose level is achieved from the variation of the intensity. It should be noted that despite an anaerobically-induced rise in blood glucose levels during exercise, protracted (night-time) hypoglycemia can occur in the post-exercise phase, and carbohydrate intake and insulin dose must be adjusted accordingly. The concrete blood glucose response of each individual, how- 


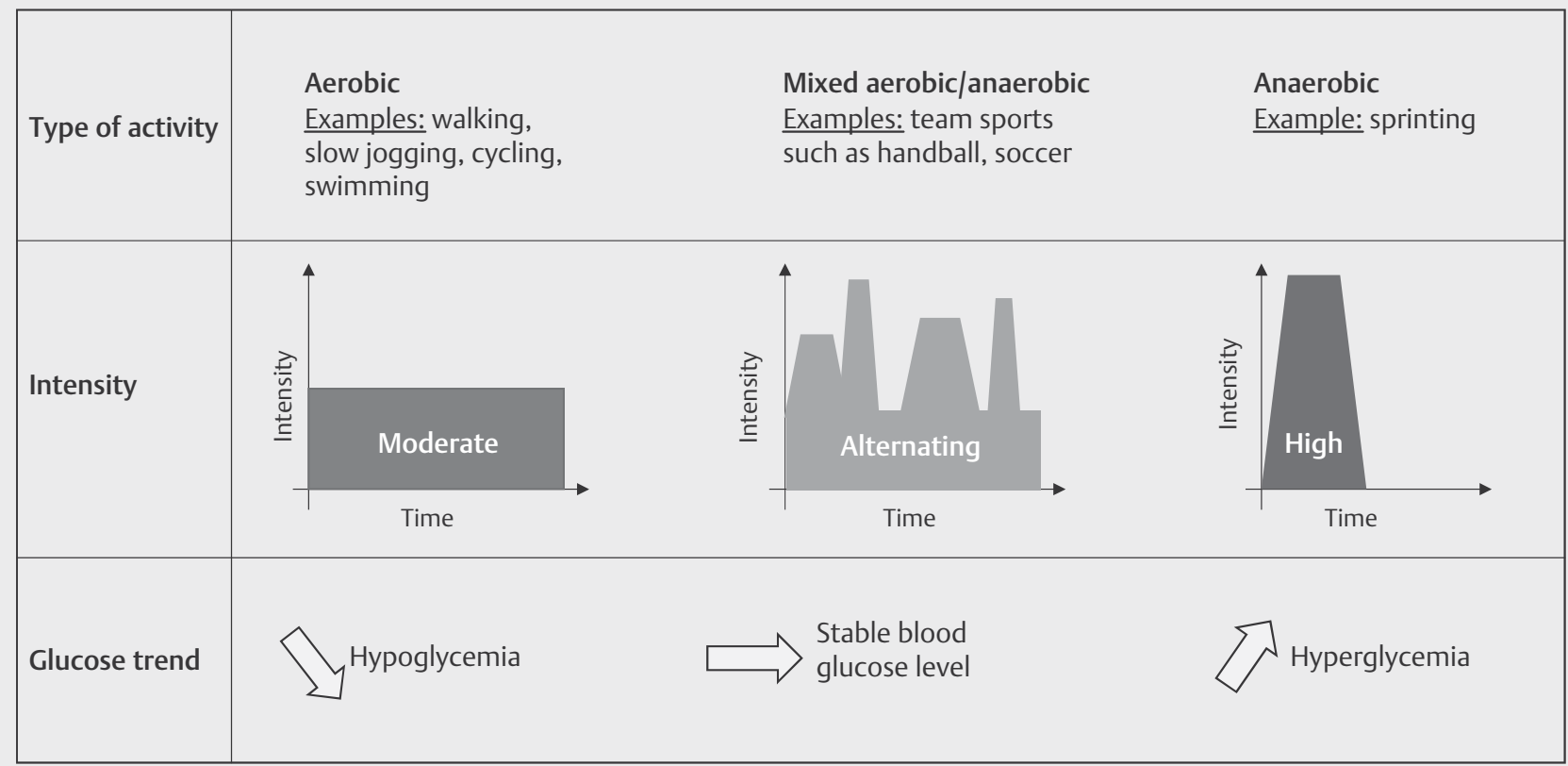

-Fig. 1 Different exercise modes and intensities (aerobic, mixed aerobic-anaerobic, anaerobic) and the respective glucose trend. Data according to [9] .

ever, depends on many factors and must be recorded using regular blood glucose measurements both during and up to $12 \mathrm{~h}$ after the activity when starting new sporting activities.

\section{Prevention of exercise-induced complications: basic rules}

- Since there are only rough dose-response relationships, individual adjustment rules must be worked out for each patient.

- For this purpose, blood glucose profiles should be created regularly during sporting activities and recorded in a sports diary together with information about insulin dose, time interval between injection and training, additional carbohydrates and form of exercise (endurance, strength training, intensity, training pulse). This protocol forms the basis for the analysis of individual metabolic reactions during sports, serves to gather experience and helps to optimize therapy with the medical or diabetes team.

- Blood glucose levels > $13.9 \mathrm{mmol} / \mathrm{l}(250 \mathrm{mg} / \mathrm{dl})$ and ketonemia (blood acetone $>1.1 \mathrm{mmol} / \mathrm{l}$ ) and ketonuria (acetone in urine) are associated with a severe insulin deficiency. This must be corrected by insulin substitution before muscular activity is started or continued.

- The use of continuous glucose monitoring (CGM) devices can be helpful to better assess metabolic reactions during/after exercise. However, the glucose level is measured in the tissue fluid (interstitium) and not in the blood. Before starting sports activities, the alarm profile in the CGM system should be adjusted: raise low alarm limit and set pre-alarm to very sensitive, raise high alarm limit or even deactivate it and set pre-alarm here to low sensitivity or deactivate it.
- When using an AID system (AID = Automatic Insulin Dosing, i.e. an algorithm reduces or increases the insulin delivery of the insulin pump depending on the CGM data), the adaptation of therapy to physical activity is very system- and product-specific. For sporting activities, AID systems usually provide the possibility of a temporary increase in the glucose target value. This must be entered in a timely manner (at least 1-2 hours before the start!); however, for longer and/or more intensive activities, this is often not sufficient. In this case, it is often recommended to pause the AID mode and to adjust the therapy manually as described in order to prevent hypoglycemia (in the AID mode, excessive insulin delivery may also occur in the "Sport Mode", e. g. after glucose increase due to sport carbohydrate unit).

- Ideally, exercise testing using an ergometer, including lactate testing or cardiopulmonary exercise testing, should be performed to determine the aerobic and anaerobic metabolism. Based on the determination of metabolic thresholds and the corresponding pulse frequencies, specific training recommendations can be made.

- Aerobic activity primarily leads to hypoglycemia, anaerobic exertion to hyperglycemia. In this last, intensive form of exercise, catecholamines are released to a greater extent, which lead to an increase in blood glucose during exercise but can particularly promote hypoglycemia in the post-exercise phase, especially 6 hours later. Night-time hypoglycemia in particular must be taken into account.

- Always carry an emergency sports set (e. g. dextrose, glucose gels, soft drinks, fruit juice) with you when doing sports in order to be able to react immediately in case of hypoglycemia.

- A CGM system should be used for longer exercise sessions performed in water, which makes blood glucose measurements impossible. In this case, the receiver of the CGM system must 
be brought along with a waterproof but radio-permeable protective cover.

- Inform sports colleagues, friends, trainers, teachers about hypoglycemia risk and countermeasures.

- Environmental conditions (heat, cold) and altitude must be taken into account as they influence the interaction between insulin, glucose, glucagon and catecholamines. In these cases, more frequent blood glucose monitoring is indicated.

\section{Dose determination for insulin and additional carbohydrates}

The following factors must be considered when determining insulin dose reduction and additional carbohydrates during exercise [8-13]:

- Age

- Cardiovascular risk factors and diseases

- Diabetes complications

- Medication

- Training status

- Type, intensity and duration of muscular activity

- Strength training lowers the glucose level in the post-exercise phase more than endurance training.

- Influences on insulin availability by ambient temperature, injection site and time of insulin injection, type of insulin (normal insulin, basal insulin, mixed insulin, insulin analogues), level of insulin dose, type of therapy (conventional insulin therapy (CT), intensified conventional insulin therapy (ICT) continuous subcutaneous insulin infusion (CSII))

- Current blood glucose level before exercise: initial values of 120-180 mg/dl are optimal.

- Time of last meal before exercise

- Type and amount of carbohydrates ingested

- Reduce normal and basal insulin by up to $50 \%$ before exercise lasting several hours or all-day, as the replenishment of muscular glycogen depots can continue until the following day.

- Carbohydrates should also be ingested after exercise.

- When doing sports of short duration and low intensity, only ingest additional carbohydrates (so-called sports-carbohydrate unit)

- Reduce bolus insulin by $25-75 \%$ when exercising during the action time of the bolus insulin

- If it is not possible to reduce the insulin dose, additional carbohydrates in small amounts (0.5-1.5 carbohydrate units) of a high energy density must be drunk or eaten every 20-30 min.

- Several servings of 0.5-1.5 carbohydrate units should be spread over the period before, during and after exercise.

- Depending on duration and intensity, additional carbohydrates of $8 \mathrm{KHE} \approx 5$ carb units or more may be required.

Suitable choices include cola, fruit juices, muesli bars, fruit and bread.

- To reduce the risk of hypoglycemia during prolonged exercise, short sprints before, during or after exercise are recommended, as short intensive intervals increase blood glucose levels for a short time. However, it must be taken into account that this intensive exertion can lead to a protracted reduction in blood glucose levels.

- Always make sure that sufficient liquid is ingested. Increased blood glucose levels require an additional increase in fluid intake to avoid dehydration.

\section{INFO BOX 1}

Prevention of sport-induced complications during therapy with an insulin pump

- For the reduction of meal bolus insulin and for additional carbohydrates the same applies as with traditional insulin injection therapy

- In case of exercise lasting more than 1-2 h, halve the basal rate for normal insulin $2 \mathrm{~h}$ and for analog insulin approx. 1 $\mathrm{h}$ before the start of exercise, then depending on duration and intensity

- If the pump is taken off for more than $2-4 \mathrm{~h}$, change to traditional insulin therapy, reduce basal rate by $10-50 \%$ up to $14 \mathrm{~h}$

\section{INFO BOX 2}

Prevention of sport-induced complications during therapy with insulin analogues

- At the start of exercise up to $3 \mathrm{~h}$ after insulin injection and a meal, reduce short-acting insulin analogues by $25-75 \%$

- At the start of exercise more than $3 \mathrm{~h}$ after insulin injection and a meal, no reduction of short-acting insulin analogues, ingest additional carbohydrates if required

- No reduction of long-acting insulin analogues during short-term exercise; if necessary, additional carbohydrates or reduction of short-acting insulin to avoid hypoglycemia

- Reduce long-acting insulin analogues (glargine) by 20-40\% before full day physical activity and 10-20\% afterwards

\section{Special note on competitive and extreme sports in type 1 diabetes}

Patients with type 1 diabetes can basically do any kind of sport, including competitive and high-performance sports. However, sports that increase the risk of impaired consciousness or impaired judgment due to hypoglycemia (e.g. diving, parachuting, extreme climbing, high-altitude ski touring, white-water canoeing or hang-gliding) are not suitable. If these sports are performed at all, they require many years of personal experience extremely cautious behavior, individual planning and intensive training.

\section{Benefits and Challenges in the Context of Physical Activity in Impaired Glucose Tolerance/Type 2 Diabetes}

\section{Pathophysiology}

The basic pathophysiological disorder in patients with impaired glucose tolerance and type 2 diabetes mellitus is impaired insulin 
sensitivity, often associated with a relative reduction in pancreatic insulin secretion. Causes are lack of exercise and a hypercaloric diet. Measures that induce an increase in insulin sensitivity, such as regular muscular exercise, offer a causal therapeutic option. Every movement of the muscle fibers increases the transmembrane glucose transport by stimulating the translocation of glucose transporters, such as GLUT-4, from intracellular locations to the muscle cell membrane. This leads to blood glucose reduction under physical exertion, a process that is not mediated by the insulin receptor and independent of the regular glucose uptake pathway [6]. This acute process, which can already be observed after 20-30 minutes, can be made permanent by constantly repeating muscular activity in endurance or strength training. Other mechanisms are also involved, such as stronger insulin binding to muscular insulin receptors, an increase in the number of muscular insulin receptors, as well as increased activity of energy metabolism enzymes and an increase in muscular capillary density [6].

\section{Therapeutic benefit}

- Lifestyle measures including a change in diet and increased physical activity to reduce weight are essential for overweight patients with type 2 diabetes and can lead to remission of the disease. Overweight diabetes patients who are cared for in a normal family doctor setting can significantly lose weight and achieve a remission of diabetes by reducing meal calories (hypocaloric meal replacement over 3 months with $836 \mathrm{kcal} /$ day) followed by a return to a normal diet and the recommendation of an optimum activity level of 15000 steps per day [14]. In a randomized intervention study, almost half of the patients (46\%) had no diabetes after one year of intervention and almost a quarter of the patients ( $24 \%$ ) had achieved a weight loss of $\geq 15$ $\mathrm{kg}$. This positive data was also confirmed in the long-term evaluation after 2 years [15]: Almost one third of the patients were still in remission. Conclusion for the practice: Lifestyle measures for weight reduction should be seen as an essential treatment for overweight patients with type 2 diabetes.

- In existing type 2 diabetes, the combination of regular endurance and strength training has the greatest effect on HbA1c levels. On average, reductions of $0.8-0.9 \%$ in addition to drug therapy are expected $[16,17]$.

- The cardiovascular risk factor profile resulting from insulin resistance and metabolic syndrome can be significantly improved by lifestyle changes [18, 19].

- The effects of lifestyle changes on the cardiovascular event rate are unclear. Macrovascular effects could not be improved in obese type 2 diabetes patients, while microvascular complications were clearly reduced $[18,20]$.

- The positive effects of physical exercise on muscular glucose transport only last for a few days after the end of training. To achieve a long-term positive therapeutic effect, exercise of at least $3 \times /$ week is necessary. A lifelong conversion to an active lifestyle with regular exercise is necessary.

\section{Obstacles on the way to an active lifestyle}

A fundamental problem is that the majority of all patients with type 2 diabetes are over 60 years of age and are unable to participate in exercise programs, or only to a limited extent, due to increased morbidity and risk factors (coronary heart disease, heart failure, peripheral arterial occlusive disease, peripheral diabetic neuropathy, proliferative diabetic retinopathy, arterial hypertension), immobility and lack of motivation. However, the benefits of physical activity are particularly evident in these cases.

- Exercise programs should be used primarily to awaken the joy of exercising and to enable people to change to an active lifestyle.

- In order to be successful with older people, exercise programs must take into account their physical abilities, age-related performance degradation, illness-related impairments, personal interests, social ties and, above all, their lifestyle.

- Concrete recommendations in terms of a training plan are essential. This should specify the type as well as the duration and intensity of exercise. It is important at the beginning that recommendations for sports and, if necessary, initial exercise instructions are given by the treating physician or diabetes nurse. This increases the credibility of exercise therapy as an essential part of the overall therapy.

- Exercise programs are doomed to fail if they physically and mentally overtax the participants. This is of central importance especially at the beginning of the training within the first 6 weeks, because in most cases the exertion level is extremely low and only covers a few $100 \mathrm{~m}$.

- The decisive factor at the beginning of the training is regularity, even if it is only 5 minutes a day. Increasing the duration and intensity is secondary in the first weeks and can be adjusted individually. As a rough rule of thumb an increase in duration of 1 min per training unit per week can be assumed (principle: "start low - go slow").

- Exercise programs that do not take these principles into account are more likely to cause harm if they trigger organic damage and awaken or intensify feelings of inferiority or guilt.

- Exercise programs can help build up new circles of friends, where hiking, Nordic walking, cycling, swimming, gymnastics or other endurance sports are cultivated. This community strengthens adherence to the programs.

- Exercise programs for patients with diabetes are also offered within the framework of outpatient diabetes groups but can also be integrated into outpatient cardiac sports groups. The latter are particularly useful for diabetes patients with heart disease.

\section{Opportunities and possibilities of the digital world in the context of movement}

The use of continuous glucose monitoring with and without insulin pump therapy has enabled many patients to better monitor, control and optimize metabolic control during exercise and sports. The individual blood glucose response to various levels of physical exertion is easier to learn, and hypoglycemia can be predicted and treated earlier [21-24].

Sensors that record non-invasive physiological signals such as heart rate, acceleration, heat flow and skin moisture can be used to monitor training [25]. Wearables (e. g. sports watches, fitness and activity trackers, smart watches) offer the opportunity to motivate and monitor individual physical activities even when the activity level is low. 
Various health apps for lifestyle optimization can be used for prevention and therapy [26-29]. There are health apps for selfmanagement in gestational diabetes $[27,30]$ or smartphone apps for athletes with type 1 diabetes [31]. Their quality and benefits should be scientifically proven and only then should they be generally recommended.

Telemedical coaching [32], digital self-help [33] and fitness portals for training via streaming or download can promote physical activity without being tied down locally. The digital world offers the opportunity to improve physical and cognitive performance, especially among children who are not physically active, by using digital movement games (exergaming) [34].

E-bikes give people with limited physical abilities new degrees of freedom and their fitness can increase when using them [35].

\section{Practical procedure for the implementation of exercise programs}

- Endurance-oriented exercise programs are primarily designed to increase aerobic capacity and are a good way to train thanks to their positive metabolic and cardiopulmonary effects. Endurance sports are recommended that allow dynamic demands on the largest possible muscle groups against the lowest possible resistance in a rhythmically constant form, such as Nordic walking, fast walking, mountain hiking, ski touring, swimming or cycling.

- Moderate endurance training (about 50-60\% of the maximum heart rate, aerobic training range) is primarily recommended in terms of training intensity. Greater effects can be particularly induced with a mix of different exercise intensities between approx. $60-80 \%$ of the maximum heart rate (mix of aerobic and anaerobic intensity) as in interval training $[16,36]$.

- Strength training for the large muscle groups can also be (additionally) recommended, as strength training leads to an increase in muscle mass and a reduction in fat, resulting in improved insulin resistance. During the training sessions, however, the risk of an increase in blood pressure in the presence of arterial hypertension should be taken into account. The blood pressure values must be optimally adjusted with medication both at rest and under exertion before the start of training (medical check-up with an exercise ECG). Care should be taken during strength training to ensure even breathing, as exhaling on exertion can cause high blood pressure peaks. For strength training, we recommend a whole-body strength endurance training program with 2-3 sets per muscle group with 15-20 repetitions and an intensity of approx. 50-65\% of the 1-repetition-maximum (RM) and, for advanced patients, a muscle-building program with 8-12 repetitions and an intensity of approx. $70-80 \%$ of the $1-\mathrm{RM}$ [37].

- Exercise programs should also include forms of activity that help improve dexterity, speed, reaction, coordination and flexibility, e. g. in ball games or dancing. Balance can also be improved by whole-body vibration training.

- Initial studies indicate that additional alternative forms of training such as electrical muscle stimulation training (EMS training), whole-body vibration training or sports with interactive computer consoles (exergaming) can also achieve positive effects in patients with type 2 diabetes, including those on glycemic values, when properly supervised [38-40], although the effectiveness compared to classic endurance/ strength training must be evaluated in future studies. Currently, diabetes mellitus is considered a contraindication for EMS training in the commercial setting [41].

- In addition, everyday activity (climbing stairs, walking, gardening, etc.) should be increased. This can also already have positive effects on glucose metabolism/insulin resistance; however, these effects are less than with targeted exercise programs.

\section{INFO BOX 3}

Principles of exercise programs in type 2 diabetes

- Heart rate is used as an indirect measure for the assessment of physical exertion, as there are reliable relationships between heart rate and physical performance $\left(\mathrm{VO}_{2} \max \right)$.

- The training pulse should be determined individually, optimally by exercise testing using an ergometer, including lactate testing or cardiopulmonary exercise testing, otherwise determination with the help of the Karvonen formula $\left(H R_{\text {train }}=H R_{\text {rest }}+\left(H R_{\text {max }}-H R_{\text {rest }}\right) x\right.$ factor 0.6). Rules of thumb, such as $H R_{\text {train }}=180 / \mathrm{min}$ - age are not suitable.

- The initial intensity and duration (initially < 10 min per training unit) should be kept low. Instead, exercise should be performed as many days a week as possible (ideally daily) or preferably short sessions several times a day. The exertion duration and intensity should be increased slowly over weeks.

- To achieve the desired long-term effects, exercise intervals of 20 min 6-7 x/week are optimal.

- In addition, the increase in everyday activity can strengthen positive effects.

\section{Avoiding complications resulting from exercise pro- grams}

- Older people with type 2 diabetes have a higher cardiovascular risk compared to healthy people of the same age. The lower the cardiopulmonary performance, the higher the risk. In order to avoid undesirable cardiovascular events after years of inactivity, a cardiological examination including maximum exercise testing using an ergometer (no age-equivalent values should be chosen as a termination criteria; instead, clear termination criteria should be selected so that an individual maximum exercise intensity can be determined) must be carried out before starting. At the same time, lactate testing or cardiopulmonary exercise testing can help to determine the optimal training intensity and to better structure individual training.

- Patients with type 2 diabetes often have cardiovascular complications such as coronary heart disease, heart failure or 
peripheral arterial occlusive disease. The intensity of training should be adapted and is limited by the underlying condition.

- In patients with type 2 diabetes, limitations in exercise capacity are often caused by diastolic heart failure (heart failure with preserved ejection fraction, HFpEF). This must be clarified before planning the training program and the training must be adjusted accordingly.

- Patients with diabetes should generally train at low-threshold levels even in exercise programs. "Running/Walking without panting" is the most important rule also for patients with heart disease. A doctor's presence is not necessary for the trained patient. A patient who recently had a heart attack should train in a cardiac rehab group under medical supervision.

- To avoid hypoglycemia, blood glucose monitoring should be performed in diabetic patients under insulin therapy before, during and after the exercise program. Adjustments to the insulin dose and additional carbohydrates should be considered.

- In proliferative retinopathy, avoid blood pressure rises above 180-200/100 mmHg. No physical exertion for 6 weeks after retinal laser or eye surgery. Strength training and fighting sport are unsuitable and potentially harmful in retinopathy.

- In peripheral diabetic neuropathy, there is a risk of manifesting a diabetic foot ulcer through unsuitable footwear.

- In case of diabetic foot syndrome, the adapted exercise program must prevent tissue overload and should be supervised by specialized trainers. In case of a diabetic foot ulcer, the exercise program must be paused.

- In case of autonomous neuropathy, the disturbance of the physiological blood pressure and heart rate regulation must be taken into account.

\section{Conflict of Interest}

MH received lecture fees from Almased, Abbott, Roche, Amgen, AstraZeneca, Berlin-Chemie, BMS, Daiichii-Sankyo, MSD, Novartis, Pfizer, Recordati-Pharma. Funding for Institution (studies): Almased for ACCORH study, Novartis for Activity Study. CB states that he has received research support/consultant fees from Astra-Zeneca, Medicap, Abbott and Muco Pharma over the past 3 years.

\section{Literature}

[1] Pedersen BK, Saltin B. Exercise as medicine - evidence for prescribing exercise as therapy in 26 different chronic diseases. Scand J Med Sci Sports 2015; 25 Suppl. 3: 1-72

[2] Kirchner H, Osler ME, Krook A et al. Epigenetic flexibility in metabolic regulation: Disease cause and prevention? Trends Cell Biol 2013; 23: 203-209

[3] Benatti FB, Pedersen BK. Exercise as an anti-inflammatory therapy for rheumatic diseases-myokine regulation. Nat Rev Rheumatol 2015; 11 : 86-97

[4] Fiuza-Luces C, Garatachea N, Berger NA et al. Exercise is the real polypill. Physiology 2013; 28: 330-358

[5] American Diabetes Association. Standards in medical care in diabetes - 2020. Diabetes Care 2020; 43: S1
[6] Stanford KI, Goodyear LJ. Exercise and type 2 diabetes: Molecular mechanisms regulating glucose uptake in skeletal muscle. Adv Physiol Educ 2014; 38: 308-314

[7] Herbst A, Kordonouri O, Schwab KO et al. Impact of physical activity on cardiovascular risk factors in children with type 1 diabetes: A multicenter study of 23251 patients. Diabetes Care 2007; 30: 2098-2100

[8] Tonoli C, Heyman E, Roelands B et al. Effects of different types of acute and chronic (training) exercise on glycaemic control in type 1 diabetes mellitus: A meta-analysis. Sports Med 2012; 42: 1059-1080

[9] Riddell MC, Gallen IW, Smart CE et al. Exercise management in type 1 diabetes: A consensus statement. Lancet Diabetes Endocrinol 2017; 5: 377-390

[10] Roberts A], Taplin CE. Exercise in youth with type 1 diabetes. Curr Pediatr Rev 2015; 11: 120-125

[11] Pivovarov JA, Taplin CE, Riddell MC. Current perspectives on physical activity and exercise for youth with diabetes. Pediatr Diabetes 2015; 16: 242-255

[12] Bally L, Laimer M, Stettler C. Exercise-associated glucose metabolism in individuals with type 1 diabetes mellitus. Curr Opin Clin Nutr Metab Care 2015; 18: 428-433

[13] Garcia-Garcia F, Kumareswaran K, Hovorka R et al. Quantifying the acute changes in glucose with exercise in type 1 diabetes: $A$ systematic review and meta-analysis. Sports Med 2015; 45: 587-599

[14] Lean ME, Leslie WS, Barnes AC. Primary care-led weight management for remission of type 2 diabetes (DiRECT): an open-label, cluster randomised trial. Lancet 2018; 391: 541-551

[15] Lean ME], Leslie WS, Barnes AC. Durability of a primary care-led weightmanagement intervention for remission of type 2 diabetes: 2 year results of the DiRECT open-label, cluster randomised trial. Lancet Diabetes Endocrinol 2019; 7: 344-355

[16] Röhling M, Herder C, Roden M et al. Effects of long-term exercise interventions on glycaemic control in type 1 and type 2 diabetes: a Systematic Review. Exp Clin Endocrinol Diabetes 2016; 124: 487-494

[17] Umpierre D, Ribeiro PA, Kramer CK et al. Physical activity advice only or structured exercise training and association with HbA1c levels in type 2 diabetes: A systematic review and meta-analysis. JAMA 2011; 305: 1790-1799

[18] Wing RR, Bolin P, Brancati FL et al. Cardiovascular effects of intensive lifestyle intervention in type 2 diabetes. N Engl J Med 2013; 369: 145-154

[19] Knowler WC, Fowler SE, Hamman RF et al. 10-year follow-up of diabetes incidence and weight loss in the Diabetes Prevention Program Outcomes Study. Lancet 2009; 374: 1677-1686

[20] Look AHEAD research group et al. Effect of a long-term behavioural weight loss intervention on nephropathy in overweight or obese adults with type 2 diabetes: a secondary analysis of the Look AHEAD randomised clinical trial. Lancet Diabetes Endocrinol 2014; 2: 801-809

[21] Colberg SR, Laan R, Dassau E et al. Physical activity and type 1 diabetes: time for a rewire? J Diabetes Sci Technol 2015; 9: 609-618

[22] Jayawardene DC, McAuley SA, Horsburgh JC et al. Closed-loop insulin delivery for adults with type 1 diabetes undertaking high-intensity interval exercise versus moderate-intensity exercise: A randomized, crossover study. Diabetes Technol Ther 2017; 19: 340-348

[23] Breton MD, Chernavvsky DR, Forlenza GP et al. Closed-loop control during intense prolonged outdoor exercise in adolescents with type 1 diabetes: the artificial pancreas ski study. Diabetes Care 2017; 40: $1644-1650$

[24] Dovc K, Macedoni M, Bratina N et al. Closed-loop glucose control in young people with type 1 diabetes during and after unannounced physical activity: a randomised controlled crossover trial. Diabetologia 2017; 60: 2157-2167 
[25] Ding S, Schumacher M. Sensor monitoring of physical activity to improve glucose management in diabetic patients: A review. Sensors 2016; 16: 589

[26] Lunde P, Blakstad Nilsson B, Bergland A et al. The effectiveness of smartphone apps for lifestyle improvement in noncommunicable diseases: systematic review and meta-analyses. J Med Internet Res 2018; 20: 1-12

[27] Wu X, Guo X, Zhang Z. The efficacy of mobile phone apps for lifestyle modification in diabetes: systematic review and meta-analysis. JMIR Mhealth and Uhealth 2019; 7: e12297

[28] Yom-Tov E, Feraru G, Kozdoba M et al. Encouraging physical activity in patients with diabetes: intervention using a reinforcement learning system. J Med Internet Res 2017; 19: e338

[29] Jimenez G, Lum E, Car J. Examining diabetes management apps recommended from a Google search: Content analysis. JMIR Mhealth and Uhealth 2019; 7: e11848

[30] Pais S, Parry D, Petrova K et al. Acceptance of using an ecosystem of mobile apps for use in diabetes clinic for self-management of gestational diabetes mellitus. Stud Health Technol Inform 2017; 245: 188-192

[31] Kordonouri O, Riddell MC. Use of apps for physical activity in type 1 diabetes: current status and requirements for future development. Ther Adv Endocrinol Metab 2019; 10: 1-7

[32] Thomas JG, Bond DS, Raynor HA et al. Comparison of smartphonebased behavioral obesity treatment with gold standard group treatment and control: A randomized trial. Obesity 2019; 27: 572-580

[33] Schütte L. Digital self-help. Digitalization and Technology Report Diabetes. 2019; https://www.dut-report.de/wp-content/uploads/2019/01/Selbsthilfe_Schuette.pdf. Access 26.05.2019
[34] Staiano AE, Beyl RA, Guan W et al. Home-based exergaming among children with overweight/obesity: A randomized clinical trial. Pediatr Obes 2018; 13: 724-733

[35] Cooper AR, Tibbitts B, England C et al. Potential of electric bicycles to improve the health of people with type 2 diabetes: A feasibility study. Diabet Med 2018; 35: 1279-1282

[36] Karstoft K, Winding K, Knudsen SH et al. The effects of free-living intervalwalking training on glycemic control, body composition, and physical fitness in type 2 diabetic patients: A randomized, controlled trial. Diabetes Care 2013; 36: 228-236

[37] Yang Z, Scott AC, Mao C et al. Resistance exercise versus aerobic exercise for type 2 diabetes: A systematic review and meta-analysis. Sports Med 2014; 44: 487-499

[38] van Buuren F, Horstkotte D, Mellwig KP et al. Electrical myostimulation (EMS) improves glucose metabolism and oxygen uptake in type 2 diabetes mellitus patients - results from the EMS study. Diabetes Technol Ther 2015; 17: 413-419

[39] Robinson CC, Barreto RP, Sbruzzi G et al. The effects of whole body vibration in patients with type 2 diabetes: a systematic review and metaanalysis of randomized controlled trials. Braz J Phys Ther 2016; 20: 4-14

[40] Kempf K, Martin S. Autonomous exercise game use improves metabolic control and quality of life in type 2 diabetes patients - a randomized controlled trial. BMC Endocr Disord 2013; 13: 57

[41] Kemmler W, Weissenfels A, Willert S et al. Recommended contraindications for the use of non-medical WB-electromyostimulation. German Journal for Sports Medicine 2019; 11: 278-281 